\title{
The Pathway towards Social Responsibility in Italian Wine Sector: The Feudi di San Gregorio S.p.A. Experience
}

\author{
Daniela Sica ${ }^{1}$, Ornella Malandrino ${ }^{1}$, Benedetta Esposito $^{1}$, Stefania Supino ${ }^{2}$ and Maria Rosaria Sessa ${ }^{1}$ \\ 1. Department of Business Sciences-Management \& Innovation Systems (DISA-MIS), University of Salerno, Fisciano (SA) 84084, \\ Italy \\ 2. University of San Raffaele, Rome 00166, Italy
}

\begin{abstract}
The traditional business view-which assumes that the main contribution of companies to society is the creation of economic value - is being surpassed by a growing awareness of other values, including social and environmental ones. The diffusion of Corporate Social Responsibility (CSR) in all sectors and, in recent time, also in the wine business, has been based on a stakeholder approach. Most of the existing key concepts and tools addressing CSR issues have been developed by, and in, the context of large enterprises. The multitude of Italian wine SMEs (small and medium-sized enterprises) remain at an early stage of environmental and social management, limited in most cases to local activities, characterized by an occasional approach and unrelated to the business strategy. But, due to increased pressure from stakeholders, environmental and social concern is gradually growing in SMEs as well. Nevertheless, CSR initiatives developed by large companies frequently fail when they are adopted by SMEs and also by the wine SMEs. In recent years, major companies have felt the need to work on CSR and progressively defined and adopted a wide range of instruments such as codes of conduct, process standard, environmental management standard, cause related marketing, corporate social reports, sustainability reports, etc. These instruments more than often represent the final result of experiments established by large sized organizations, often operating in distant countries, which have decided to satisfy the multiple requests from the always more complex context in which they operate. Many companies, therefore, have orientated themselves towards the accomplishment of new objectives centred, not only on gaining profit but, moreover, towards the creation of values: a more extensive value better known as "pluri-dimensional" shared by all the stakeholders within a new interdependent dynamism and inspired by a multitude of references (such as UN Global Compact, AA1000 Guidelines, Global Reporting Initiative, SA8000, etc.). The perception of the concept of corporate responsibility and its possible practical application in a wine-growing company is the subject of continuous debates, always in search of a globally shared interpretation. Sustainable and social initiatives require, in fact, planning, monitoring and enhancement of knowledge. This is a process that requires a tirelessly oriented approach to continuous improvement. The aim of this paper is to delineate efficient ways of promoting CSR, considering wine SMEs' specific profiles and needs. Moreover, the paper is aimed at highlighting case of Feudi di San Gregorio S.p.A. The company has always been oriented to pursue management models capable of generating social value (in conjunction with that economic) at the systemic level (so-called shared value). This in order to evaluate the contribution that CSR instrument adopted has offered to build organization's sustainability in its three dimensions: economic, environmental and social.
\end{abstract}

Key words: Corporate social responsibility, value creation, socio-environmental tools, ethical code.

\section{Introduction}

The progressive extension of "social responsibility" to the business world is defining a challenging role for

Corresponding author: Daniela Sica, Ph.D., research fields: circular economy, sustainable management and corporate social responsibility. the business community from which a rethinking of the same goals that businesses are called to pursue. In fact, alongside the traditional production function, which still represents the constitutive element of their being and operations, they are asked to drive to sustainability, in the context of enhancing economy, human and nature. 
Similarly, stakeholders are now more involved in taking behaviors in line with the principles of sustainability and, above all, acquiring propositional and coherent attitudes in relevant processes for the purpose of concrete social innovation. Due to the central role of the stakeholders, a reinterpretation of social responsibility is being drawn up, from corporate social responsibility to company stakeholder responsibility, through an approach that favors sustainability as a correct, fair and transparent form of value creation for all stakeholders.

Therefore, the need has emerged to improve information flows on performance and socio-environmental organization, in the awareness that the traditional accounting documents of an economic nature have significant limits in accounting for the multiplicity of intangible assets such as reputation, trust, and consensus. In fact, although they make a significant contribution to the process of creating wealth generated by the company, they do not find adequate accounting in their operating budgets.

It started a process aimed at the enlargement of the corporate information, which exceeded the now restricted, and strongly structured, the riverbed aspects connected to the balances of economic-financial capital - to involve those linked to communication on social value of business.

All stakeholders, internal and external, financial and non-financial, have expressed this requirement of accountability and will participate in a concrete and coherent, in order to promote the transition from models based on corporate social responsibility to other based on company stakeholders responsibility.

These models require, however, the involvement of SMEs (small and medium-sized enterprises), through innovative approaches to social responsibility, which do not involve the dissemination of practical "standard", but promote an act of undertaking capable of successfully combining responsibility and competitiveness.

The aim of this paper is the critical analysis of the evolutionary dynamics that characterized the concept of corporate social responsibility in order to scan a best practice of the wine Italian SMEs.

Therefore, the work is articulated into four sections. Initially, the difficulties of SMEs in the wine sector were analyzed in adopting the tools for Corporate Social Responsibility (CSR). Wine SMEs are often characterized by a concrete social commitment, albeit unrecognizable, as it is characterized by an almost "intimate" declination, an expression of the entrepreneur's ideal thrust, who realizes social interventions by considering expressions of his own sphere rather than that of your organization.

However, the involvement of wine SMEs, and in general of all SMEs, is needful for the "building" of lasting sustainability as it is capable of integrating growth in competitiveness, environmental protection and social development. This is because of the extraordinary synergies that may arise from the structured adoption of CRS-marked routes and the promotion of local development-oriented dynamics. Therefore, we analyzed the management tools for CSR adopted by an Italian SMEs: the Feudi di San Gregorio S.p.A., in order to explore the opportunities and the main elements of criticality that derive from a strategic drive towards improving their performance in a holistic approach.

In the final section we outline the main considerations, highlighting the main outcomes of the study.

\section{The Socio-Environmental Dimension in Wine SMEs}

The established CSR concept requires organizations to pursue socially-qualified economic goals by implementing integrated management of the different aspects of cost-effectiveness, environmental protection, improvement of employment conditions and equity and social cohesion.

Taking social responsibility is a complex concept that is constantly evolving and therefore difficult to operate. 
The actions, models and tools for CSR are varied and, as we have seen, ranging from ethical codes to environmental management systems, from ethical certification to social marketing to various types of socio-environmental reporting tools [1]. They are essentially designed for large companies, often operating in more than several countries, and therefore more exposed to stakeholder pressures on environmental and social performance.

This set of tools is therefore hardly adoptable at SMEs which constitute the backbone of the productive fabric of the current enlarged Europe and, as is well known, of our country.

In the Italian reality, however, compared to developed European economies, SMEs are characterized by peculiar elements of characterization, among which the average, in terms of number of employees, more reduced, the relative financial vulnerability as well as the prevailing structure of family type.

The involvement of SMEs is therefore indispensable for the "building" of lasting sustainability, as it is capable of integrating growth in competitiveness, environmental protection and social development. This is in consideration of the extraordinary synergies that may arise from the structured adoption of CSR-led pathways and the promotion of dynamic local development.

However, a careful analysis of national and international dynamics shows that the orientation of entrepreneurial organizations towards social responsibility reflects inevitably the company size. Indeed, the adoption of the relevant instruments is really small among SMEs, in particular among those wine, while, on the contrary, represents a prerogative of larger organizations.

The reasons for this are due to a multitude of reasons, among which it is worth highlighting that wine SMEs, on the one hand, show little knowledge of the practices and tools that can be adopted and, on the other, perceive an excessive difficulty. In fact, the proper implementation of social responsibility pathways requires specific skills and high organizational capacity, coupled with a strong focus on adequate and incisive communication policies: all these factors, generally absent in smaller organizations.

These factors must be flanked by other, including the limited availability of financial resources, which makes wine SMEs little inclined to make plans and investments with returns in the medium-long term, as moreover requires the realization of correct and effective social responsibility strategies and structured involvement and lasting of stakeholders.

However, although it is an aspect still little explored from a theoretical point of view, the investigations to the analysis of behavior and socio-environmental wine SMEs in Italy have highlighted a discreet attention in respect of aspects linked to CSR.

Indeed, such analyses, based on real behavior towards the main stakeholders rather than the formal adoption of the instruments, affirm that the socio-environmental commitment is not a far-reaching or marginal element for wine SMEs and that they often are socially responsible. This is because, in the face of known weaknesses, wine SMEs have strong points, consisting of true and own peculiarities able to facilitate the path oriented toward a Triple Bottom Line Approach [2].

SMEs have a strong and immediate recognition of the entrepreneur's figure and its central role in making decisions, flexibility and ease of adaptation to changes in the context of reference, interpersonal relationships characterized by broad involvement with strong emphasis on human values and of the person, due to the widespread ability to develop a plot of positive relationships according to informality typical of small contexts.

They are substantially constituted by a strong attention to the needs of employees and those of the territory, which often comes from the value system that drives the entrepreneur-owner, or the family of reference in the case of companies' family. 
Moreover, the deep roots in the local socio-economic context of wine SMEs allow them a close link between enterprise and territory, recalling the modus operandi previous to the phenomenon of globalization, a link that feeds informal relationships and forms of mutual help. Productive districts also often have a social value, which transforms them into areas of development of technical and commercial knowledge, entrepreneurial culture, educational and training structures, services to workers and their families.

It is thus necessary to delineate innovative approaches, designed for wine SMEs, wherein the CSR should be interpreted as company stakeholders responsibility in order to avoid the diffusion of "standard practices" and to promote "corporate behaviors" capable of effectively combining responsibility and competitiveness [3].

They will have to provide information and awareness-raising, able to put in light of the potential and reflections on the competitive performance. This creates a stable, systematic and planned link between socio-environmental commitment, stakeholder engagement, and enhancement of communication.

Sometimes it is the low propensity to communicate, especially to the outside, which obscures social engagement in wine SMEs making them "operators silent” of CRS.

Greater dissemination, transparency and communication of good practices and socially responsible behaviors could represent an important multiplier, capable of generating a sort of "domino effect" in a context such as that of wine SMEs, where imitation and re-interpretation are important ways of managing and organizing innovation.

Particularly fruitful could be the adoption of network-based approaches or forms of collaboration between groups of undertakings at sectoral level or of territorial area or district, suitable to grasp the economies of scale and identifying real priorities for local intervention [4]. In fact, in the case of small and very small organizations, the socio-environmental impact of the single actor is often insignificant, but becomes very significant when considering the district as a whole.

In this scenario, although the benefits in terms of image would be less as shared among the enterprises involved, even costs - the embodiment, discovery, monitoring, reporting-would become more sustainable, due to the distribution between the different actors concerned.

Attention to socio-environmental aspects could become, for district businesses, an important lever of qualitative differentiation, capable of enhancing the wealth of intangible resources. Among them are the highest level of staff motivation, reputation among the social partners and, more generally, a renewed image made in Italy, where the social commitment represents a key factor in competitiveness and differentiation.

\section{The CSR in the Italian SMEs: The Feudi di San Gregorio S.p.A. Experience}

Feudi diSan Gregorio S.p.A. group is made up of companies operating in the wine sector ${ }^{1}$, which in a holistic way adopt the principles of quality, transparency, fairness, professionalism and hospitality on which the business modus operandi is based [5].

${ }^{1}$ Affect the Group:

- The wine cellar of Feudi di San Gregorio S.p.A., the core business of the Group, and today, a symbol of the winemaking renaissance of southern Italy.

- The Manduria Estate, born from the dream of enhancing the Puglia vineyards.

- The Cefalicchio Estate, located in Puglia, is biodynamic.

- San Gregorio Refreshment from which Marennà emerges, a refined Michelin starred restaurant expresses the constant search for a seemingly obscure equilibrium between innovation and tradition, in perfect harmony with the group vision.

- Comete Field, acquired in 2016, located in the very prestigious area of the Bolgheri Doc in Tuscany.

- Basilisk, acquired in 2011 and situated on the slopes of Vulture and with organic management. It is the result of a recovery project of the ancient lava grottoes in the "Shesh".

- Sirch, of which the Group has a stake in the Sirch family mission to rediscover the traditional style of Friulian wines, children of respect for the land and the almost exclusive exploitation of vineyard work. 
The vision, globally shared, is born from the goal of spreading a wine culture in Italy and the world, oriented to the valorisation of the territory where wine traditions and innovation, history and the future coexist and synergistically evolve.

Feudi di San Gregorio S.p.A. has a hybrid configuration, a mixture of mechanical bureaucracy, adhocracies and simple structure.

In particular, an organization with a top-down power flow was identified by the line organs, an inadequate support component, and a highly-engineered, operationally-engineered staffing system, continuous interaction and value co-organization.

The organizational culture of Feudi of San Gregorio S.P.A. is constructive. The doctrine identifies the general lines of this type of organizational culture by defining specific rules and for each of them the relative organizational characteristic.

There emerges within the group the coexistence of two types of subculture:

- strengthening, married by employees at the birth of the company characterized by a more fervent adherence to the basic values of the dominant culture;

- orthogonal, married by the employees recruited with the advent of the new presidency, characterized by an adherence to the basic values of dominant culture contemplated at different and specific, but not conflicting values.

The coexistence of tradition and innovation, reinforcing and orthogonal subculture, makes Feudi di San Gregorio a company able to grasp the needs of the market in its entirety.

In recent decades, the pressures emerging from the competitive arena in which the company Feudi di San Gregorio S.p.A. operates, have led management to redefine their approach, strategies, and organizational planning in order to achieve better economic, environmental and ethical performance.

The Feudi di San Gregorio has therefore assumed precise economic and moral responsibilities, based on socially shared ethical principles, in response to a series of instances from the community and the territory, as well as from the institutional and economic context. Of course, in a symmetrical way, it has begun over time to progressively develop and subsequently adopt quality and social quality management programs.

In this perspective, the Feudi di San Gregorio S.p.A. has implemented the ISO 22000 and the Product Certifications (e.g. BRC and IFS).

Only recently, it has been equipped with tools of social responsibility to create a true good corporate citizenship, designed to provide guarantees, protection and support for the well-being of the community in which he operates.

The corporate management, in fact, aware of the impacts of its activities on society and the environment, has tried to achieve a proper integration between the company's economic goals and social goals through voluntarily and consciously adopted policies. Feudi di San Gregorio S.p.A. acknowledges that the responsibilities arising from the exercise of its activity go beyond the traditionally considered players, i.e. management, shareholders and creditors, to include a wider circle of stakeholders, including employees, suppliers, consumers and society in general, i.e. all those who in various capacities are affected by the consequences of corporate policies.

Therefore, the management has rethought the corporate strategies in a socially responsible way. It has therefore taken initiatives that enable the organization to strive towards sustainable development through a trade-off between economic performance and social performance. This is to internalize and make the organization's social dimension tangible through careful consideration of the expectations of community members and the identification of objectives compatible with stakeholders' interests.

It has therefore defined appropriate procedures for the selection, qualification and monitoring of suppliers and subcontractors, based on their ability to comply with the requirements of the standard.

This is in order to activate a virtuous circle, aimed at 
involving a growing number of companies in the “ethical path”.

The Feudi di San Gregorio S.p.A. envisaged the involvement of civil society in the process of monitoring the behavior of the company. This is through interviews with local organizations, trade union representatives and other stakeholders, both in the phase of obtaining certification and in the periodic monitoring of its maintenance.

\subsection{The CSR in Feudi di San Gregorio S.p.A.: Operational Steps}

The path to transforming business ethics into a concrete CSR program has been marked by some particularly significant moments, outlined below.

3.1.1 Integration of Social Goals into the Company’s Mission

Management has deliberately chosen to include social and ethical values among the goals to be pursued, regardless of any economic benefits that this may entail.

It is in this way that aspects of the environment, the institutions, the centrality of the person and human rights, or the transparency of information and financial transactions are the subject of risk management strategies.

To this end, an appropriate management training program on CSR has been implemented at this stage, which has provided the knowledge required to handle the issues in a conscious, organic and planned way. Given the continuous evolution of the subject, there are plans to organize periodic updating programs to maintain its modern and innovative strategies.

3.1.2 Formal Recruitment of Social Responsibility and Enlargement of Risk Management Strategies

Management is aware that social commitment is not a point of arrival but, above all, a starting point that is realized when the pursuit of ethical, social and environmental values is officially consecrated in the acts defining the modalities and aims of the activity. To this end, Feudi di San Gregorio S.p.A. has developed, as the main instrument of self-regulation and implementation of ethical-social responsibility, the ethical code.

With the preparation of the ethical code, Feudi di San Gregorio S.p.A. has defined and outlined the behavioral guidelines and the values that are inspired by its relationships with all stakeholders, both internal and external.

It has introduced a clear and explicit definition of the ethical and social responsibilities of each participant in the organizational structure of stakeholder groups, laying the foundations for a fair and effective management of transactions and human relations, and supporting the reputation to create a climate of trust and mutual cooperation.

A crucial role played, therefore, is constant commitment assumed by Feudi di San Gregorio S.p.A. promoting the knowledge of the ethical code by all the members of the organization and other stakeholders who directly or indirectly interact with it. From constructive dialogue with the various stakeholders, in fact, a process, through listening to the instances and balancing them with the company's strategies, aims to activate a virtuous circle aimed at continuous improvement of its performance.

Feudi di San Gregorio S.p.A. provided for the establishment of a supervisory body (the Ethics Committee) with powers of initiative and control, with the task of monitoring, through appropriate procedures, the operation and compliance of the code, as well as ensuring that you upgrade.

The system of implementation and control of the ethical code of Feudi di San Gregorio S.p.A. also envisages the identification of an ethics officer who has the operational responsibility of the corporate ethics program and educates the Ethics Committee-which is hierarchically dependent-executes its decisions and informs it of its activities.

Feudi di San Gregorio S.p.A. also uses an internal ethical auditing, an independent and objective activity of assurance and support, aimed at improving the 
efficiency and efficiency of the organization.

The implementation of ethical code of conduct through concrete management tools thus becomes a further statement of the real will of the organization to take on the concerns of the stakeholders and to meet ethical and social values that were, primarily addressed to the global community, will end in the long run representing a real added value and also an economical type for the enterprise.

\subsubsection{Identifying Stakeholders}

Once integrated, even formally, social commitment within the goals to be achieved, it was necessary to implement it first in favor of those individuals, entities or social groups that can be made part of the notion of stakeholders. Since the stakeholder concept has led to a fairly wide-ranging approach, at this stage, corporate management first identified in a more detailed way by defining a stakeholder map, which is the most likely to have the negative consequences of the activity of the company (Fig. 1).

With respect to that community, Feudi di San
Gregorio S.p.A. has implemented appropriate policies for containment and prevention of social costs, and established transparent forms of dialogue and involvement with their representatives.

The behaviors that characterize the modus operandi of each participant in the organizational structure of Feudi di San Gregorio S.p.A., in fact, are based on the observance of the law, regulations, and statutory provisions, codes of self-discipline, ethical integrity and fairness in a framework of transparency, honesty, good faith and full respect for the competition rules. In particular, the growth strategy of Feudi di San Gregorio S.p.A. is based, in particular, on the following values:

- Transparency

In the conduct of its relations Feudi di San Gregorio S.p.A. provides its interlocutors with clear, complete and timely access to useful information to correctly and transparently interpret the economic, social and environmental impacts of their business activities.

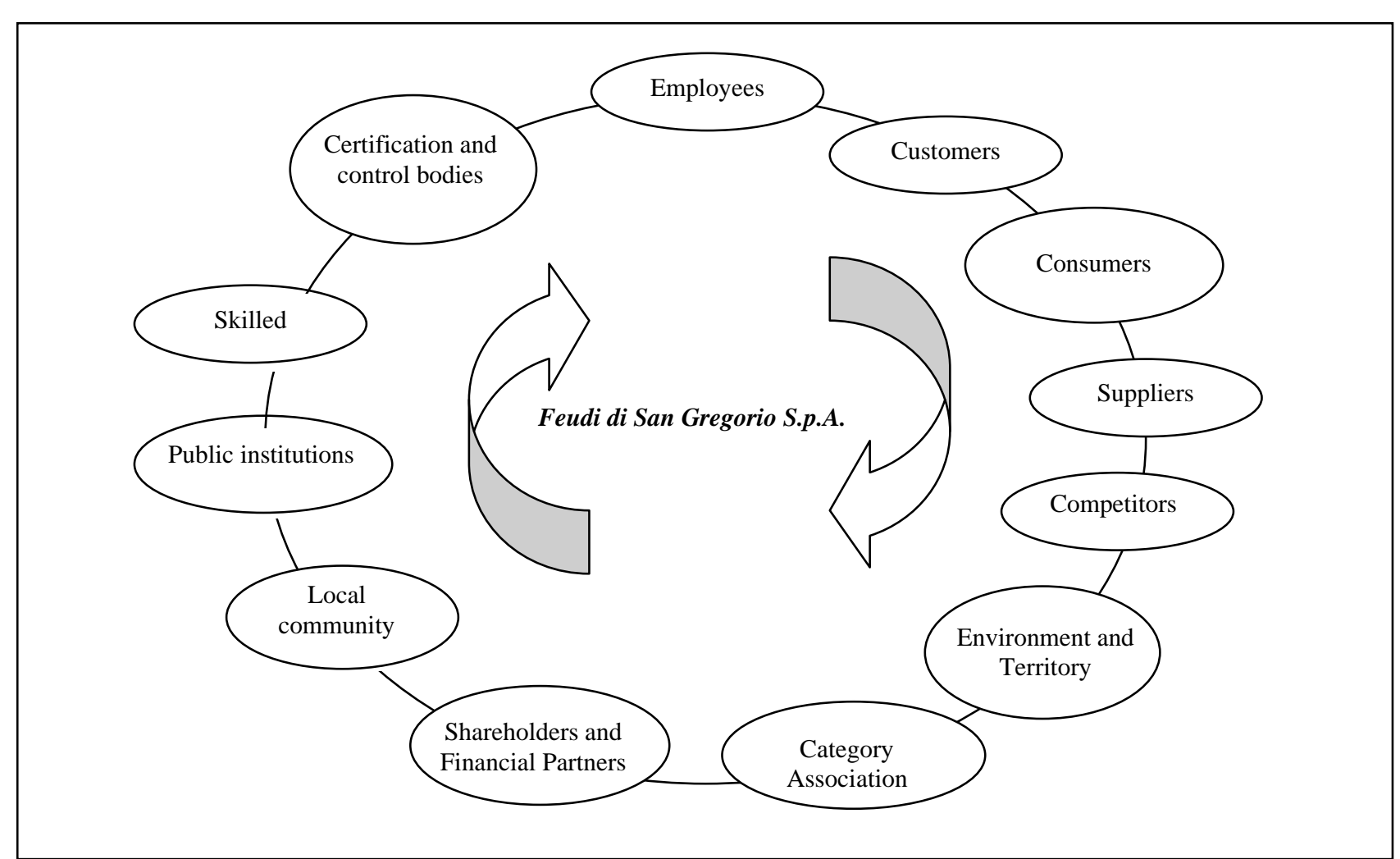

Fig. 1 Stakeholders map of Feudi di San Gregorio S.p.A. 


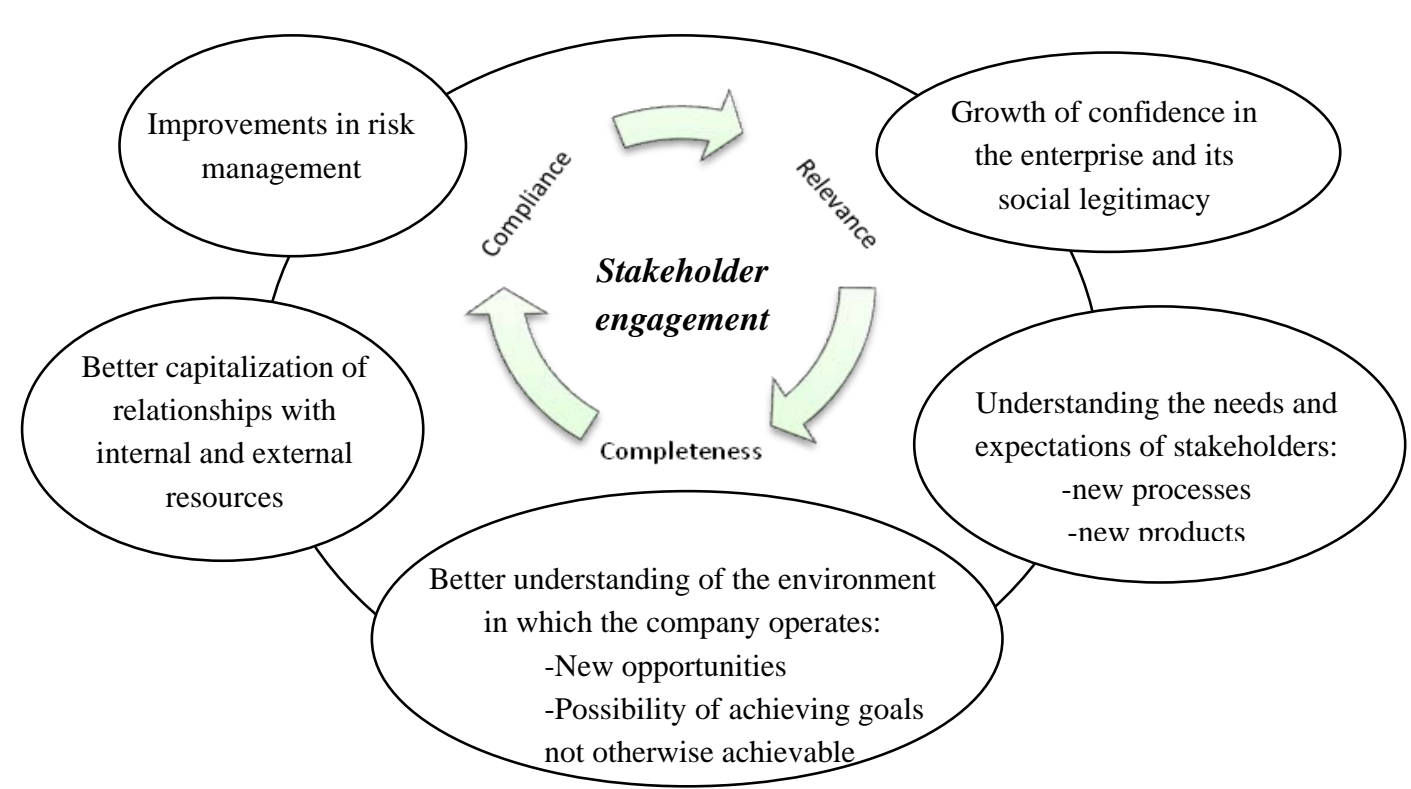

Fig. 2 Stakeholder engagement in Feudi di San Gregorio S.p.A.: main benefits.

\section{- Correctness}

All the actions, operations and negotiations carried out and, in general, the behaviors put in place by the members of the organization in the course of the work are inspired by the utmost fairness, honesty, clarity and legitimacy in the formal aspect and substantial.

\section{- Consistency}

Feudi di San Gregorio S.p.A. adopts and maintains over time a carried out in line with the values, the mission and the principles of operation of the company. They are considered to be the foundation of strategic planning, objectives and operational management and also contribute to determining its corporate identity and entrepreneurial philosophy.

Similarly, under the same conditions, Feudi di San Gregorio S.p.A. prefers the interlocutors who, in their behaviors, are consistent with their values.

\section{- Professionalism}

All Feudi di San Gregorio S.p.A. activities must be carried out with professional commitment and rigor, respecting the roles and responsibilities assigned, in order to protect the prestige and reputation of the company. The goals of the organization, the proposal and the implementation of projects, investments and actions are aimed at increasing the company's assets, management, technology and knowledge values, as well as the creation of value and well-being for all stakeholders in the long term [6].

Bribery, illegal favors, collusive behavior, stress, direct and/or third party, personal and career benefits for themselves or others are without exception prohibited.

Feudi di San Gregorio S.p.A. uses multiple tools for stakeholder engagement ranging from on-line consultations to individual meetings and/or interviews, as well as specific surveys to check the concerns of certain stakeholder groups on some critical aspects.

Identifying stakeholders as recipients of formalized safeguards in codes of conduct and providing accurate risk management tools for the pursuit of social goals brings with it a clear awareness of the value and benefits of a business-oriented policy prevention (Fig. 2). This is to assess ex ante and avoid pipelines that can also cause high social costs, such as environmental or occupational safety, where appropriate measures can prevent or minimize the risk of accidents. In addition, the responsibility for implementing an internal control system and an 
effective risk management system is common at every level of the organizational structure of Feudi di San Gregorio S.p.A. As a result, all members of the organization, within the functions and responsibilities covered, are committed to defining and actively participating in the proper functioning of the internal control and risk management system.

\subsubsection{Internal and External Communication}

Feudi di San Gregorio S.p.A. introduces its internal and external stakeholders to their principles and behavioral norms by advertising the ethical code through its own website and through specific communication activities (such as delivery to all employees internal and external copies of a copy of the code at the time of the hire or start of the collaboration relationship or the inclusion of an informative note on the adoption of the code in all contracts, etc.).

In order to ensure the correct understanding of the values and principles set out in the ethical code for all employees, Feudi di San Gregorio S.p.A. provides a training activity aimed at creating a sharing on the contents of the code itself, also providing tools for awareness and knowledge of mechanisms and procedures to translate ethical principles into behaviors to be held concretely in day-to-day operations.

In particular, the training initiatives are implemented with modalities and content appropriate to the role played in the company from each collaborator and developed according to a path to complete vocational training and enhance personal development.

\section{Conclusion}

The paper highlights that currently there is still space to introduce best practices in a systematic way and to adopt the correct CSR instruments in wine SMEs. Moreover, these must become an integrated part of the managerial strategies, in reference to the internal and external dimension of companies.

In reference to the internal dimension, possible interventions principally regard management of human resources (in terms of continuous education and formation, flexibility of working hours, equal opportunities) and environmental management (in terms of energy saving, co-generation, emission reductions, use of recycled materials).

In reference to the external dimension, the relationship with the main local communities, commercial partners and suppliers is taken into consideration.

It is essential to support wine SMEs in the acquisition of knowledge to valorise social commitment. That is feasible through the creation of a stable, systematic and planned connection between socio-environmental commitment and the search for visibility on what has been created. The scarce attention of communication towards the outside often hides, today, wine SMEs' social commitment, characterizing them as "silent operators" of CSR.

Moreover, for wine SMEs to acquire competence and capacity in terms of CSR they should promote forms of collaboration and exchanges of experience, perhaps with large-sized companies which have already established significant knowledge and know-how in the field of CSR.

\section{References}

[1] Supino, S., and Sica, D. 2011. "Nuovi paradigmi di rendicontazione d'impresa: il report integrato.” Esperienza d'Impresa 2: 81-91.

[2] Proto, M., and Supino, S. 2009. Dal Management Ambientale alla Reponsabilità Sociale delle Organizzazioni. Stato dell'arte e dinamiche evolutive. Giappichelli Ed. Torino.

[3] Molteni, M., and Todisco, A. 2007. Piccole e Medie Imprese e CSR. La CSR come leva di differenziazione. CSR Manager Network Italia.

[4] Citterio, A., and Lenzi, I. 2005. "Reti di territori, reti di responsabilità sociale." In Rapporto sullo sviluppo sostenibile, 8.

[5] Feudi di San Gregorio. Tra vino e arte. Accessed 16.01.13. https://www.feudi.it/.

[6] Cheng, B., Ioannou, I., and Serafeim, G. 2014. "Corporate Social Responsibility and Access to Finance." Strategic Management Journal 35 (1): 1-23. 